investigate the effect of pulmonary artery systolic pressure (PASP) to the response of patients with CRT.

Methods 93 patients (76 male, mean ages: $61.23 \pm 15.56)$ with heart failure involved in our study. According to the level of preoperative PASP, they were divided into three groups (Group I: PASP $\geq 50 \mathrm{~mm} \mathrm{Hg}$, $\mathrm{n}=29$; Group II: $30 \mathrm{~mm} \mathrm{Hg} \mathrm{0.05)}$ and Group II (from $69 \mathrm{~mm}$ to $66 \mathrm{~mm}, \mathrm{p}>0.05)$. LVEF increased averagely by $31-38 \%(\mathrm{p}<0.01)$ in Group III at 3-6 months, But in Group I and II which had not obvious increased at 3 months ( $p>0.05)$ and had increased by $17 \%$ (Group I, $\mathrm{p}<0.05$ ) and 26\% (Group II, $\mathrm{p}<0.01$ )at 6 months. LVEF in Group III had increased more than that in Group I and Group II $(\mathrm{p}<0.05-0.01)$.

Conclusions Clinical outcomes post-CRT can be predicted by elevated PASP. A preoperative PASP $\geq 50 \mathrm{~mm} \mathrm{Hg}$ is associated with increased risks for adverse events and a higher mortality for aggravation of heart function.

\section{e0554 ORS DURATION CHANGES DURING DIFFERENT VV INTERVALS AFTER CARDIAC RESYNCHRONISATION THERAPY}

\section{doi:10.1136/hrt.2010.208967.554}

Danfang Li, Donghong Wang, Hongyun Zang, Haibo Yu, Yaling Han. Shenyang Northern Hospital

Introduction The aim of this study was to investigate the influence of different VV intervals on ORS duration of surface ECG after cardiac resynchronisation therapy (CRT).

Methods 54 patients after CRT treatment due to congestive heart failure (CHF) in our hospital were enrolled in this study, of which 43 cases with the ORS duration of surface ECG $>120 \mathrm{~ms}$ and 11 cases with QRS duration $\leq 120 \mathrm{~ms}$ (Tissue doppler ultrasound showed the existence of ventricular asynchrony), all patients were appropriate for CRT or CRT-D implantation with type I or IIa indication. After CRT implantation, the VV durations were programed by the pacemaker programme at 9 different settings: simultaneous left and right ventricle pacing, left ventricle preexcitation (left ventricle+20, 40,60 and $80 \mathrm{~ms}$, respectively), and right ventricle pre-excitation (right ventricle+20, 40, 60 and $80 \mathrm{~ms}$, respectively). During these VV intervals, the aortic velocity time integral (VTI) was measured by echocardiography, and we defined the VV intervals with the highest VTI as the best VV interval, ORS duration of surface ECG was recorded at the same time.

Results There is no significant difference of ORS durations among different VV intervals. In the simultaneous pacing of LV and RV model, the best VV interval was $12.96 \%$ of all the 54 patients, it was $37.02 \%$ in left ventricle pre-excitation and $49.98 \%$ in right ventricle pre-excitation. This was suggested that sequential pacing of the two ventricles was superior to the synchronously pacing. There was no significant correlation between the best VV interval and the width of QRS wave $(r=0.205, p=0.136)$, and the width of QRS wave were the smallest in 9 patients $(9 / 54,16 \%)$ in the best VV interval, but that of the other 45 patients were not the smallest, so it was inaccurate for assessing the best VV interval merely by the width of ORS wave, and we'd better to optimise VV intervals with the help of echocardiography.

Conclusions There were no significant changes of the ORS durations during different VV intervals after CRT treatment. The sequential pacing of the two ventricles could bring $87 \%$ patients better haemodynamic effects. There was no significant correlation between the best VV interval and the width of ORS wave, and the width of ORS duration of most patients in the best VV interval was not the smallest. The effect of CRT could be improved more by optimisation of the VV interval individually.

\section{e0555 AN 5-YEAR FOLLOW-UP OF PATIENT WITH VALVULAR HEART DISEASE AND CHRONIC ATRIAL FIBRILLATION UNDERGOING ABLATION OF THE ORIFICES OF THE PULMONARY VEINS UNDER DIRECT VISION BY USING THE TRANSBALLOON ULTRASOUND ABLATION CATHETER}

doi:10.1136/hrt.2010.208967.555

Li Li Zhi-Yun Xu, Yong Cui. Department of Cardiothoracic Surgery, Changhai Hospital, Second Military Medical University, Shanghai, China

Objective To evaluate the effect and possibility of surgical ablation of the pulmonary vein orifices under direct vision with transballoon ultrasound ablation catheter for patients with permanent atrial fibrillation and rheumatic valve disease.

Methods 21 consecutive patients with rheumatic valve disease and permanent atrial fibrillation undergoing mitral valve replacement surgery from December 2002 to September 2003 were enrolled for this study. All cases were divided into 2 groups by whether or not receiving an additive pulmonary vein ablation procedure. The test group ( 6 male, 5 female, aged $51.55 \pm 7.83$ years, atrial fibrillation duration $5.50 \pm 5.40$ years, left atrial diameter $7.27 \pm 1.39 \mathrm{~cm}$, LVEF $53.95 \pm 4.54 \%$ and NYHA class II-IV) undertook a surgical isolation of the pulmonary vein orifices by using a transballoon ultrasound ablation catheter addition to routine mitral valve replacement. The control group ( 3 male, 7 female, aged $53.30 \pm 7.86$ years, atrial fibrillation duration $4.50 \pm 3.47$ years, left atrial diameter $6.74 \pm 0.62 \mathrm{~cm}$, LVEF $56.91 \pm 3.78 \%$ and NYHA class II-IV) received the valve replacement surgery alone.

Results There were not any complications in both groups. With an electrical cardioversion 3 months after the surgery, $73 \%$ patients in the ultrasound ablation group were free from AF over 1 year while only $10 \%$ patients in control group $(\mathrm{p}=0.003)$. During an average follow-up duration of $69.92 \pm 4.61$ months, $65.6 \%$ were in sinus rhythm in ultrasound ablation group while none in the control group. Left atrial volume decreased significantly at 5 year after surgery compared to 3 months after surgery in the test group $\left(78.83 \pm 32.39 \mathrm{~cm}^{3}\right.$ VS $\left.150.78 \pm 52.32 \mathrm{~cm}^{3} \mathrm{p}<0.05\right)$, and the end systolic diameter (LAESD) and end diastolic diameter? (LAEDD) also decreased $(3.92 \pm 0.43 \mathrm{~cm}$ vs $5.09 \pm 0.98 \mathrm{~cm}, \mathrm{p}<0.05$; and $3.92 \pm 0.43 \mathrm{~cm}$ vs $4.46 \pm 1.15 \mathrm{~cm}, \mathrm{p}<0.05$, respectively).

Conclusions Ablation of the orifices of the pulmonary veins under direct vision with transballoon ultrasound ablation catheter during mitral valve surgery is effective to maintain sinus rhythm after electrical cardioversion and can be performed safely. The function of left atrial and cardiac output improves during long term follow-up.

\section{E0556 CATHETER ABLATION OF ATRIAL TACHYCARDIA FROM THE NONCORONARY CUSP OF THE AORTIC VALVE}

doi:10.1136/hrt.2010.208967.556

Zhou Xianhui, He Li, Taang Baopeng, Long Deyong, Li Jinxin, Zhang Yu, Li Yaodong. Department of the Echocardiograph, First Affiliated Hospital, Xinjiang Medical University, Urumqi

Objective In this study, we examine the characteristics of anteroseptal Atrial tachycardias (AT) originating from the Noncoronary Cusp (NCC) of the Aortic Valve, and demonstrate the long-term efficacy and safety of targeting the arrhythmias by Catheter Ablation.

Methods From among a cohort of 43 patients with symptomatic focal AT undergoing electrophysiological evaluation, the point of earliest activation was at NCC region in 7 patients.

Results The arrhythmia terminated with $<10$ seconds of radiofrequency delivery and was successfully eliminated in 7 patients. All

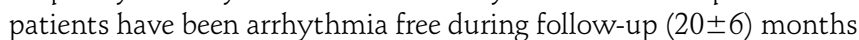

